\title{
Risk of preterm birth after treatment for cervical intraepithelial neoplasia among women attending colposcopy in England: retrospective-prospective cohort study
}

\begin{abstract}
Objective To explore the association between preterm delivery and treatment at colposcopy.

Design Retrospective-prospective cohort study using record linkage.

Setting 12 National Health Service hospitals in England.

Participants Women who had a cervical histology sample taken between 1987 and 2009. These women were linked by hospital episode statistics to hospital obstetric records between 1998 and 2009 for the whole of England to identify singleton live births between $20-43$ gestational weeks before or after cervical histology.

Main outcome measures Proportion of preterm births ( $<37$ weeks); the relative risk for the strength of association between preterm births and treatment for cervical intraepithelial neoplasia.

Results 18441 singleton births occurred: 4176 before histology and 14 265 after histology. Of the singleton births after histology, $9.0 \%(n=1284)$ were preterm compared with $6.7 \%$ of all births in England over the same period (excess risk 2.3 per 100 births, 95\% confidence interval 1.8\% to $2.8 \%$ ). Among first births after histology, the adjusted relative risk associated with previous treatment was 1.19 (95\% confidence interval 1.01 to 1.41 ); among first births before histology the relative risk associated with subsequent treatment was 1.47 (1.05 to 2.05). Combining these, the relative risk associated with treatment adjusted for timing relative to histology was 0.91 ( 0.66 to 1.26 ) corresponding to an absolute difference of -0.25 ( -2.61 to 2.11 ) per 100 singleton births. Among 372
\end{abstract}

women who gave birth both before and after treatment, there were 30 preterm births after treatment and 32 before treatment (relative risk 0.94 , 0.62 to 1.43 )

Conclusion The risk of preterm delivery in women treated by colposcopy in England was substantially less than that in many other studies, predominantly from Nordic countries. The increased risk may be a consequence of confounding and not caused by treatment. Although this study is reassuring for large loop excision of the transformation zone overall, it is possible that deep conisation or repeated treatment leads to an increased risk of preterm delivery.

\section{Introduction}

Screening for cervical cancer aims to detect and treat cancer precursors (cervical intraepithelial neoplasia) before progression to invasive cancer. Women with abnormal cytology are referred to colposcopy for further assessment. In the United Kingdom, most of these women will either have no procedure (and be discharged) or have a punch biopsy sample taken at their first colposcopy appointment to confirm the presence or absence of disease, whereas others (particularly those with high grade cytological abnormalities) may be offered excisional treatment at the first visit. ${ }^{1}$ Those with a histological sample showing high grade cervical intraepithelial neoplasia are commonly treated by large loop excision of the transformation zone (LLETZ). Other less common types of fertility preserving treatment include 
laser conisation, cold knife excision, cold coagulation, and other ablative treatments. ${ }^{2}$

In recent years several studies have linked treatment for cervical intraepithelial neoplasia with a higher risk of subsequent preterm delivery (before 37 completed weeks of gestation). Preterm infants are associated with substantial emotional and economic costs to their families and communities and have a disproportionate impact on health service utilisation. In 2006 a widely cited meta-analysis of 27 included studies found that large loop excision of the transformation zone was associated with preterm delivery, low birth weight, and preterm premature rupture of membranes. ${ }^{3}$ Since then several studies have been published on this subject. The largest to date, a Norwegian record linkage study of 57136 births before treatment and 15 108 after treatment, found the proportion of preterm deliveries in each group, respectively, to be $6.7 \%$ and $17.2 \% .{ }^{4}$ Few studies are from the United Kingdom and those that are tend to be generally small ${ }^{5-9}$ and do not confirm the strong association between treatment and subsequent preterm delivery found elsewhere. Colposcopy and treatment of cervical intraepithelial neoplasia in England is quality assured by the National Health Service cervical screening programme and self regulated by the British Society for Colposcopy and Cervical Pathology..$^{10}$ It is possible that the results reported internationally are not representative of large loop excision of the transformation zone as carried out by specially trained colposcopists working to detailed clinical guidelines.

We explored the association between preterm delivery and treatment at colposcopy by comparing three populations: external (population of England), internal (within the cohort), and within individual women. We reasoned that although excisional treatment might result in a subsequent preterm delivery, punch biopsy is too small to have such a causal role. Furthermore, by definition treatment cannot affect the outcome of a birth that occurred before that treatment. In our analyses we adjusted for any increased risk associated with a history of cervical intraepithelial neoplasia and focused on the association with the treatment itself.

\section{Methods}

We identified women from clinical records in 12 NHS hospitals as having had a cervical histology sample taken (by a punch biopsy at colposcopy or excisional treatment, or both) between January 1987 and December 2009. Hospitals included in the study responded to an invitation for participation in a letter posted on the British Society for Colposcopy and Cervical Pathology website and sent to the hospitals' mailing lists. A prerequisite for participation was that the units had over 350 new referrals a year and support from their local research and development office. Of units that expressed an interest we selected the largest ( $>550$ new referrals a year) in each region of the country to ensure representation from all of England. Three selected units could not obtain local ethical approval in time and we substituted these with others in regions already represented in the study and one with fewer than 300 referrals a year.

For each woman, we obtained the date of the first and last histological sample recorded in the clinic and requested the most severe procedure (that is, whether it was a punch biopsy or material from excisional treatment) carried out at these visits. Thus we split our cohort into three groups: women with a punch biopsy, women with excisional treatment, and women with a record of cervical histology but missing treatment status. We followed these women prospectively (through retrospective linkage) for gestational age of subsequent births, and retrospectively for gestational age of previous births.

To identify live births whether before or after the histological sample had been obtained, we linked women by their NHS number and date of birth to hospital episode statistics of inpatient obstetric records between April 1998 and April 2010 for the whole of England. The NHS number is a unique identifier issued when a birth is registered or when an individual first registers with an NHS general practitioner. Hospital episode statistics is a data warehouse containing details of all admissions to NHS hospitals in England, including private patients treated in NHS hospitals. ${ }^{11}$ From hospital episode statistics records we obtained information on month and year of delivery, gestational age, birth weight, onset of delivery, mode of delivery, resuscitation method, number of previous pregnancies, duration of stay in hospital, and any inpatient diagnosis or operation recorded for the mother.

\section{National comparison}

To obtain the proportion of preterm deliveries in the population for the study period we extracted and pooled NHS maternity statistics ${ }^{12}$ (published by hospital episode statistics) from 2000-01 to 2009-10. We were unable to find data before April 2000 and would have considered age standardised proportions, but the published reports of gestational age by maternal age did not separate singleton from multiple births as we have done here. $^{13}$

We considered only births with a known gestational age and that were between 20 and 43 weeks. As best practice indicates that pregnancies should not exceed the 42nd gestational week, we excluded infants born at gestational ages greater than 43 weeks to avoid any inaccuracies of gestational ages over 43 weeks. Because of concerns over accuracy we also excluded births with a recorded gestational age under 20 weeks (599 nationally and 16 in our cohort). We excluded multiple births (twins and triplets). The same exclusions applied to the NHS maternity statistics.

To avoid having to adjust for clustering of preterm deliveries within individuals we limited the internal analysis to the first pregnancy recorded in our cohort data during the 11 year period between 1998 and 2010. We also excluded antepartum stillbirths or stillbirths of indeterminate timing $(n=216)$ on the basis that we could not establish whether preterm delivery was induced as a result of the death of the fetus or vice versa. Supplementary table A 1 shows the distribution of births in the cohort by parity, whether the mother was treated before or after delivery, and type of treatment received.

\section{Statistical analysis}

To obtain relative risks and $95 \%$ confidence intervals for the difference in proportions of births that were preterm compared with those that were term, we used relative risk regression (an alternative to logistic regression for cohort studies) using the glm command in Stata. We adjusted the relative risks for maternal age at delivery $(<25,25-34,>34)$, parity $(0,1,2, \geq 3)$, and study site. To determine parity in the cohort we used the number of previous pregnancies recorded by hospital episode statistics, except where a birth was found in the dataset that was not reflected in the parity field. For completeness we also report the results of risk differences, also estimated using the glm command in Stata. P values cited are for the Pearson's $\chi^{2}$ test for the difference between proportions or for the likelihood ratio test in other situations. 
We compared singleton births using three groups. The first groups (external comparisons) were relative to NHS maternity statistics for the whole of England. In the external comparisons we compared all births in the cohort, all births after histology, and births after treatment with all births in England. For the internal comparisons we considered only first births in the dataset and compared (a) births after histology with those before histology, and births after treatment with each of (b) births before treatment, (c) births after histology in untreated women, and (d) births before histology in untreated women adjusting for disease history. The within woman comparison was between the last birth before colposcopy and the first after colposcopy in a given woman. We considered all women with births both before and after colposcopy and the subset of treated women. We also considered the ratio of the after to before effect in treated women relative to the effect in untreated women (that is, with biopsy only). The confidence interval for the women with biopsy only was obtained by treating the two relative risks (within treated women and within untreated women) as independent. In the within woman analyses we did not adjust for parity or maternal age. For dealing with the question of the effect of treatment on preterm delivery, we considered the most relevant analyses to be for the external comparison, births after treatment; for the internal comparison, births after treatment compared with births before histology adjusting for disease history; and for the within woman comparison, all treated women with births before and after treatment.

The risk ratio comparing births after treatment with births before histology adjusting for disease history is obtained from the interaction term ( 1 for post-treatment, 0 otherwise) in a generalised linear model with an effect for treatment ( 1 for treated or subsequently treated, 0 otherwise) and an effect for timing ( 1 for post-histology, 0 otherwise). In the absence of other adjustments, it is simply the ratio of the relative risks of preterm delivery in (a) births after treatment compared with births after biopsy only to (b) births before treatment compared with birth before biopsy only.

For comparison with published meta-analyses, we added our study to those in the published meta-analysis of 27 included studies to determine whether it differed significantly (taking into account the heterogeneity in earlier studies) using the metareg command in Stata. All analyses were done in Stata 11 (release 11.2. College Station, Texas).

\section{Results}

\section{Participating hospitals}

On average, the 12 participating clinics were similar to the other 215 clinics in England for published data on colposcopy (see supplementary table A2). With one exception, the mean value of each data item in participating clinics was within the interquartile range of the other clinics. The one exception was the proportion of biopsy results reported within eight weeks, which was $83 \%$ in the study clinics but at least $86 \%$ in $75 \%$ of other clinics nationally. The clinics included in this study accounted for $8.5 \%$ of all patients having new colposcopy in England in 2005-06. ${ }^{1}$

\section{Cohort}

A total of 44210 NHS numbers of women with data on cervical histology were submitted for linkage to hospital episode statistics, $81.3 \%$ (35 958 women) had at least one hospital admission between 1998 and 2009 and 46.8\% (16 816/35 958) of those with a record had at least one birth recorded. A total of 26897 births (in 16816 women) were identified (fig $1 \Downarrow$ ). Of these, 18441 were singleton births with known gestational age between 20 and 43 gestational weeks, of which 1616 were preterm (20-36 weeks) and 471 were very preterm (20-32 weeks). Table $1 \Downarrow$ presents the proportion of births by maternal age at delivery, parity, timing relative to colposcopy, and procedure at colposcopy.

\section{External comparison (population based)}

The average preterm delivery rate in England between 2000 and 2010 was $6.7 \%$ (34 153/510 660, fig $2 \Downarrow$ ). The preterm rate varied from $6.9 \%$ in 2000 to $5.9 \%$ in 2009 , with a minimum of $5.9 \%$ in 2009 and a maximum of $7.6 \%$ in 2004 . Overall, the observed preterm rate in our cohort was $8.8 \%$ (1616/18 441), yielding an excess risk of preterm delivery of 2.08 per 100 singleton births (95\% confidence interval $1.66 \%$ to $2.49 \%$; $\mathrm{P}<0.001)$ and a relative risk of 1.31 (95\% confidence interval 1.25 to 1.37 ) compared with the general population. The proportion of births after cervical histology that were preterm was $9.0 \%$ (1284/14 265), giving an excess risk of 2.31 per 100 births ( $1.84 \%$ to $2.79 \%)$ and a relative risk of 1.35 (1.28 to 1.42$)$ compared with the general population. Similar results were observed for deliveries after treatment $(9.4 \%, 449 / 4776$, table $2 \Downarrow)$. This relative risk $(1.41,1.29$ to 1.54$)$ was significantly $(\mathrm{P}=0.03$, even after allowing for heterogeneity in the meta-analysis) lower than the comparable result in a meta-analysis $(1.97,1.78$ to 2.17$) .{ }^{14}$ The proportion of preterm births after histology, however, varied widely by hospital (fig 2) from $6.2 \%$ (161/2608, Wirral University Teaching Hospital) to $15.6 \%$ (33/212, St Mary's Hospital, Imperial College) $\left(\chi_{11}^{2}=66.07, \mathrm{P}<0.001\right)$.

\section{Internal comparison}

For comparisons within the cohort only the first birth recorded in the dataset was included for each woman, and antepartum stillbirths and stillbirths of indeterminate timing were excluded. This left 12937 births of which 1099 (8.5\%) were preterm. Overall, 52.6\% $(n=578)$ of these preterm births had a gestational age of $35-36$ weeks, $19.3 \%(n=212)$ at $33-34$ weeks, $17.5 \%$ $(\mathrm{n}=192)$ at $29-32$, and $10.6 \%(\mathrm{n}=171)$ at $20-28$ weeks. The mean maternal age at first recorded delivery $(\mathrm{n}=12$ 937) was 31 (interquartile range 27-34) years.

Among singleton births delivered after cervical histology, $8.9 \%$ (832/9368) were preterm compared with 7.5\% (267/3569) of those delivered before the date of histology (table 2). The increase in risk of preterm delivery (adjusted by study site, parity, and maternal age at delivery) was significant (adjusted relative risk $1.32,95 \%$ confidence interval 1.13 to 1.53 ). Figure $3 \Downarrow$ plots the relative risks for all 12 study sites: there was no evidence of heterogeneity between sites $\left(\chi_{11}^{2}=11.574, \mathrm{P}=0.40\right)$. The type of sample taken at colposcopy was recorded for $80.6 \%$ of births in the cohort (10 423 singleton births) including $77.8 \%$ $(n=855)$ of preterm deliveries. Of those that had a delivery after colposcopy, the risk of preterm delivery in women who had a treatment was $9.1 \%$ (283/3095) compared with 8.3\% (396/4770) in women who had a punch biopsy only (adjusted relative risk $1.19,1.01$ to 1.41 ). The absolute increased risk of preterm delivery after treatment when compared with a biopsy only adjusted for study site, parity, and maternal age was 1.5 per 100 births $(0.1 \%$ to $2.9 \%)$.

The relative risks for treated versus punch biopsy were similar for births before the histological sample was taken (table $3 \Downarrow$ ). Of births before histology, 7.8\% (81/1045) of those in women who were subsequently treated were preterm compared with $6.3 \%(95 / 1513)$ in women who subsequently had a punch biopsy 
and no treatment (adjusted relative risk 1.31, 0.97 to 1.76, table 3 ). Thus the risk ratio comparing births after treatment with births before histology adjusting for disease history was 0.91 (95\% confidence interval 0.66 to 1.26 ).

The proportion of births that were under 33 gestational weeks (see supplementary table A3) in those women who had a histology sample taken before birth was 2.8\% (243/8779) compared with $2.0 \%$ (66/3368) in women who had a histology sample taken after birth (adjusted relative risk 1.60, 1.18 to 2.18). The adjusted relative risk in births after colposcopy comparing treatment with biopsy only was 1.23 (0.89 to 1.69$)$. Further adjustment for disease history yielded a relative risk for delivery under 33 weeks of 0.81 ( 0.43 to 1.52 ).

Since the analysis was limited to the first recorded birth in each woman, the risk ratio (treated versus punch biopsy) was also examined and was different in second or subsequent births after colposcopy compared with first births after colposcopy. The adjusted relative risk for treatment in second and subsequent births after colposcopy was non-significantly $(\mathrm{P}=0.39)$ greater than for first births after colposcopy, but similar to that of the last birth before colposcopy (see supplementary table A4).

\section{Within woman}

An analysis was carried out restricted to women who had a birth both before and after a colposcopy (within woman comparison, table 2). For each woman the last birth before treatment and the first birth after treatment was only included. This left 1078 women. There were $80(7.4 \%)$ preterm births before colposcopy and $98(9.1 \%)$ after colposcopy (relative risk $1.23,95 \%$ confidence interval 0.95 to $1.59, \mathrm{P}=0.15$ ). In 372 women with births both before and after treatment, there were 30 preterm births after treatment and 32 before treatment (the relative risk of preterm birth after treatment was $0.94,0.62$ to 1.43 ). In 501 women with births both before and after punch biopsy, the relative risk of preterm birth after a punch biopsy was $1.14(0.77$ to 1.66). The ratio of the risk ratios (of preterm birth after:before colposcopy) for treated compared with untreated (biopsy only) women was 0.82 ( 0.27 to 3.17 , see supplementary table A5).

Since in general the risk of a preterm birth is greater in a first birth than in a second birth $(8.8 \% v 7.6 \%$ in the study cohort, table 1), and given that most women who gave birth both before and after colposcopy had exactly one birth before colposcopy (so that that second births were compared with first births), the relative risk will have been underestimated. However, in women with at least two births the risk of preterm on first birth was less (8.1\%), yielding a relative risk of 0.98 (0.85 to 1.13$)$ for second birth compared with first birth in women with at least two births.

\section{Discussion}

In this study of 18441 singleton deliveries in women who had a cervical biopsy sample taken during colposcopy in England, the additional risk of a preterm birth over that in the general population was 2.1 per 100 singleton births, yielding a relative risk of 1.31. Comparing births in women within the cohort (table 2 ), the relative risk in women who previously had treatment (conisation, large loop excision of the transformation zone, loop excision) compared with those who only had a biopsy was 1.19 . However, the relative risk of preterm delivery in women before colposcopy comparing those who subsequently had treatment with those who subsequently only had a biopsy was also greater than 1 (1.33). Consequently the risk ratio comparing births after treatment with births before histology adjusting for disease history was less than 1 , with an upper limit of the $95 \%$ confidence interval of 1.26. Furthermore, in 372 women who gave birth both before and after treatment for cervical intraepithelial neoplasia, the number of births that were preterm was fewer after treatment than before (30v32).

\section{Strengths and limitations of the study}

In considering causality in the absence of a randomised controlled trial, we took into account confounding by risk modifying factors and the temporality of cause and effect. We allowed for the possibility of general confounding: factors (such as smoking or ethnicity) that might predispose a woman to both abnormal cervical cytology and preterm births; confounding by disease severity - the possibility that factors (such as immune suppression) that make it more likely for a woman to have high grade disease (and be treated by cone excision) will also make her more likely to have a preterm birth; and disease causing prematurity - that the disease in itself (or factors that lead to its presentation) rather than its treatment makes a woman more likely to have a preterm birth. In our analyses we attempted to take into account all three possible sources of confounding. The first analyses included all singleton births in the cohort to ease comparison with published population statistics. Although we present the risk of preterm birth for both the whole cohort and births after treatment, we did not adjust for possible confounding. The internal analysis eliminates general confounding because all women by definition have had colposcopy. To exclude confounding by disease severity, we considered women with treatment and compared births after treatment with those before treatment. To exclude confounding owing to disease causing preterm delivery, we compared births after treatment with those after biopsy only. To take account of both disease severity and temporality, we calculated the ratio of these relative risks. This ratio was less than 1 , suggesting that the associations observed in the other analyses could all result from confounding. Finally we took births before and after colposcopy in the same woman. Such an analysis is complicated by changing parity and maternal age, but the relative risk after treatment was less than 1 . Additionally, the post-colposcopy relative risk in treated women was less than that in women who only had a punch biopsy (see supplementary table A5) suggesting that confounding has not artificially reduced the relative risk of treatment. However, for $19.4 \%$ of women we do not know the type of procedure carried out at colposcopy. This could have an important impact on the estimates comparing treated with untreated women if, for instance, those with an unknown procedure were more likely both to be treated and to subsequently have a preterm birth. This problem will be further investigated in phase 2 of this study (a nested case-control study).

The results in this paper depend on the quality of birth data submitted by participating clinics (NHS trusts) to hospital episode statistics. The proportion of preterm births will also be affected by the population served by the clinic. For example, Whipps Cross Hospital serves a community with a high proportion of ethnic minority groups, whereas St Mary's Hospital is a referral centre for high risk pregnancies from across London.

There is also a question as to how representative the colposcopy units in this study are of colposcopy done across England. The 12 participating units included both teaching and non-teaching hospitals but were primarily self selected. We therefore investigated the extent to which they seemed to be representative of all colposcopy clinics in England on the basis of published data. Comparing nationally collected statistics from the clinics in this study with the other $(n=215)$ clinics in England showed that in terms of these statistics, the clinics in this study were not 
atypical of the rest of the country. Colposcopy clinics in England are audited every three years, as are all colposcopists to maintain their membership with the British Society for Colposcopy and Cervical Pathology. Thus the standard of colposcopy in England is likely to be more homogeneous than in many countries. Even if treatment in smaller centres resulted in a greater risk of preterm delivery, we believe that this study (with $8.5 \%$ of all new patients in England) is representative of most colposcopy in England.

We tried to minimise biases in this study by restricting the analysis to the first live singleton birth recorded for each women and by adjusting the relative risk by study centre. Additionally, the design of the study avoided recall and selection bias.

However, we had no information on risk modifying factors such as ethnicity or smoking, nor did we have any detailed information on treatment received at colposcopy.

\section{Comparison with other studies}

This is the largest study of preterm delivery in women with cytological abnormalities in the United Kingdom. A meta-analysis including 30 cohort studies in total found that the type of comparison group was important in determining the relative risk of preterm delivery. ${ }^{14}$ When the comparison group was external (such as the general population) the relative risk of preterm delivery was 1.97 (95\% confidence interval 1.78 to 2.17). Similarly, when the comparison group was internal (comparing births after treatment with those before treatment), the relative risk was 1.96 (1.46 to 2.64). However, when the analysis was carried out within a cohort of women with cytological abnormalities comparing treated with untreated women, the relative risk was 1.25 (0.98 to 1.58$)$. Three studies from Nordic countries obtained relative risks between 1.8 and 2.8 (all except one not included in the meta-analysis). Of the excluded studies, a large study from Norway found a relative risk of 2.13 (95\% confidence interval 2.06 to 2.20) comparing (all not just singleton) births after treatment with births before treatment, but reported a declining relative risk during the study period. ${ }^{4}$ The absolute risk was $17.2 \%$ (in 15108 births after treatment). Our preterm risk in treated women is clearly less. A study from Denmark had a relative risk of 2.8 (95\% confidence interval 2.3 to 3.5 ) compared with an external control group. ${ }^{15}$ The same study also provided a within woman odds ratio of 2.8 (95\% confidence interval 1.0 to 10.0) as did a study from Finland (1.8, 95\% confidence interval 1.04 to 3.21$){ }^{16}$

The relative risk of preterm delivery after treatment observed in this study compared with the population as a whole is substantially (and significantly) less than that found in the studies included in the Bruinsma meta-analysis. ${ }^{14}$ Additionally, our internal analyses tend not to support the hypothesis that treatment increases the risk of preterm delivery, by a factor of about 1.7 to 2.0; the analysis that adjusts for both the timing of the delivery relative to colposcopy and whether there was treatment or just a punch biopsy gives a relative risk of 0.91 (95\% confidence interval 0.66 to 1.26 ) for births subsequent to treatment.

Several studies have suggested that it is the amount of tissue removed from the cervix that produces the excess risk, not the procedure itself. ${ }^{17-19}$ It is possible that owing to the quality assurance of the colposcopy programme in England through both the cervical screening programme and the British Society for Colposcopy and Cervical Pathology, tissue removed during colposcopy is kept to a minimum and this could explain the smaller relative risks that we observed. This might be particularly relevant in the self selected colposcopy units in this study. We are currently undergoing phase 2 of this study in which we will attempt to obtain detailed colposcopy and pathology information on all women with a preterm delivery and a sample of women with a term delivery in this cohort. In particular, we are recording the measurements of the tissue excised and whether the woman was treated more than once. It seems likely that removal or destruction of a large amount of tissue may increase the risk of subsequent preterm delivery more than is seen on average.

\section{Conclusions and policy implications}

The results presented here are encouraging. Accepting the limitations of this study, women treated within the NHS cervical screening programme and particularly those treated in large colposcopy units should be reassured that, in this study of 44 000 women having colposcopy including 14265 singleton births after colposcopy, the risk of a birth being preterm was $9.0 \%$ and only slightly greater than the risk in the general population. Phase 2 of this study should strengthen the results presented here and provide information on the risk associated with the depth of cervical tissue removed.

Members of the PaCT Study Group were responsible for the collection of data included in this study. N Gul and A Miles (Wirral University Teaching Hospital), A Hollingworth and R Wuntakal (Whipps Cross University Hospital London), N Singh and A Parberry (Barts and the London NHS Trust), J Palmer (Royal Hallamshire Hospital, Sheffield), N Das and L Russ (Royal Cornwall Hospital), N Wood and S Preston (Royal Preston Hospital Lancashire), M Hannemann and D Fuller (Royal Devon and Exeter NHS Foundation Trust), K Lincoln and P Rolland (The James Cook University Hospital, South Tees), S Ghaem-Maghami and P Soutter (Hammersmith Hospital, Imperial College), R Hutson (St James University Hospital, Leeds), P Senguita and J Dent (North Durham County and Darlington Trust), and D Lyons (St Mary's Hospital, Imperial College).

Contributors: PS analysed the data and designed the database. He is the guarantor of the study and therefore accepts full responsibility for the work and the conduct of the study, had access to the data, and controlled the decision to publish. AC collated and analysed the data. All authors designed and established the study, wrote the paper, and approved the final version.

Funding: This manuscript presents independent research funded by the National Institute for Health Research (NIHR) under its research for patient benefit programme (No PB-PG-1208-16187). The views expressed are those of the authors and not necessarily those of the National Health Service, the NIHR, or the Department of Health.

Competing interests: All authors have completed the ICMJE uniform disclosure form at www.icmje.org/coi_disclosure.pdf (available on request from the corresponding author) and declare: no financial relationships with any organisations that might have an interest in the submitted work in the previous three years; and no other relationships or activities that could appear to have influenced the submitted work.

Ethical approval: This study was approved by the Brompton, Harefield, and NHLI research ethics committee, Charing Cross Hospital, London (No 09/H0708/65).

Data sharing: The statistical code is available from the corresponding author at p.sasieni@qmul.ac.uk.

\footnotetext{
Lancucki L, ed. Cervical screening programme, England: 2005-06. NHS Information Centre, 2006.

Luesley DLS, ed. NHS cervical screening programme. Colposcopy and programme management. Guidelines for the NHS cervical screening programme. NHS Information Centre, 2004.

3 Kyrgiou M, Koliopoulos G, Martin-Hirsch PL, Arbyn M, Prendiville W. Obstetric outcome after conservative treatment for intraepithelial or early invasive cervical lesions: systematic review and meta-analysis. Lancet 2006;367:489-98.
} 


\section{What is already known on this topic}

Most studies of preterm delivery after large loop excision of the transformation zone found that treatment was associated with increased risk

An influential meta-analysis (27 studies) found a relative risk of 1.70 (95\% confidence interval 1.24 to 2.35 )

Subsequent large studies from Nordic countries estimated the relative risk to be between 1.8 and 2.8

\section{What this study adds}

After adjusting for confounding, the increased risk of preterm delivery in births after treatment for cervical intraepithelial neoplasia ceases to exist

There is only a small chance (2.5\%) that the risk of preterm delivery is increased by more than 3.5 per 100 births in women treated in England

The relative risk here is significantly less than reported previously possibly because colposcopy treatment is quality assured

$4 \quad$ Albrechtsen S, Rasmussen S, Thoresen S, Irgens LM, Iversen OE. Pregnancy outcome in women before and after cervical conisation: population based cohort study. $B M J$ 2008;337:a1343.

5 Reilly R, Paranjothy S, Beer H, Brooks C, Fielder H, Lyons R. Birth outcomes following treatment for precancerous changes to the cervix: a population-based record linkage study. BJOG 2012;119:236-44.

6 Cruickshank M, Flannelly G, Campbell DM. Fertility and pregnancy outcome following large loop excision of the cervical transformation zone. Br J Obstet Gynaecol 1995:102:467-70.

7 Shanbhag S, Clark H, Timmaraju V, Bhattacharya S, Cruickshank M. Pregnancy outcome after treatment for cervical intraepithelial neoplasia. Obstet Gynecol 2009;114:727-35.

8 Haffenden DK, Bigrigg A, Codling BW, Read MD. Pregnancy following large loop excision of the transformation zone. Br J Obstet Gynaecol 1993;100:1059-60.

9 Tan L, Pepera E, Haloob RK. The outcome of pregnancy after large loop excision of the transformation zone of the cervix. J Obstet Gynaecol 2004;24:25-7.

10 British Society for Colposcopy and Cervical Pathology. Constitution. BSCCP, 1975

1 HES Online. What is HES? 2005-2007. 2011. www.hesonline.nhs.uk/Ease/servlet/ ContentServer?sitelD $=1937 \&$ category $\mathrm{ID}=456>$.

12 NHS Information Centre. NHS maternity statistics, 2000-2010. 2010. www.ic.nhs.uk/pubs.

13 Office for National Statistics. Gestation-specific infant mortality in England and Wales, 2009. www.ons.gov.uk/ons/rel/child-health/gestation-specific-infant-mortality-in-englandand-wales/2009/index.html.

14 Bruinsma FJ, Quinn MA. The risk of preterm birth following treatment for precancerous changes in the cervix: a systematic review and meta-analysis. BJOG 2011;118:1031-41.
15 Ortoft G, Henriksen T, Hansen E, Petersen L. After conisation of the cervix, the perinatal mortality as a result of preterm delivery increases in subsequent pregnancy. BJOG 2010;117:258-67.

16 Jakobsson M, Gissler M, Paavonen J, Tapper AM. Loop electrosurgical excision procedure and the risk for preterm birth. Obstet Gynecol 2009;114:504-10.

17 Noehr B, Jensen A, Frederiksen K, Tabor A, Kjaer SK. Loop electrosurgical excision of the cervix and subsequent risk for spontaneous preterm delivery: a population-based study of singleton deliveries during a 9-year period. Am J Obstet Gynecol 2009;201:33,e1-6.

18 Acharya G, Kjeldberg I, Hansen SM, Sorheim N, Jacobsen BK, Maltau JM. Pregnancy outcome after loop electrosurgical excision procedure for the management of cervical intraepithelial neoplasia. Arch Gynecol Obstet 2005;272:109-12.

19 Sadler L, Saftlas A, Wang W, Exeter M, Whittaker J, McCowan L. Treatment for cervical intraepithelial neoplasia and risk of preterm delivery. JAMA 2004;291:2100-6.

Accepted: 23 July 2012

\section{Cite this as: BMJ 2012;345:e5174}

This is an open-access article distributed under the terms of the Creative Commons Attribution Non-commercial License, which permits use, distribution, and reproduction in any medium, provided the original work is properly cited, the use is non commercial and is otherwise in compliance with the license. See: http://creativecommons.org/licenses/bync/2.0/ and http://creativecommons.org/licenses/by-nc/2.0/legalcode. 


\section{Tables}

Table 1/ Proportion of births by maternal age at delivery, parity, timing relative to colposcopy, and procedure at colposcopy

\begin{tabular}{|c|c|c|c|}
\hline Characteristics & Total No & $\%(\mathrm{No})$ preterm & $\%$ (No) very preterm $*$ \\
\hline \multicolumn{4}{|c|}{ Maternal age at delivery: } \\
\hline$<20$ & 395 & $8.1(32)$ & $2.5(10)$ \\
\hline $20-24$ & 2013 & $9.7(196)$ & $2.8(56)$ \\
\hline $25-29$ & 5082 & $8.7(440)$ & $2.7(136)$ \\
\hline $30-34$ & 6590 & $8.8(579)$ & $2.5(162)$ \\
\hline $35-39$ & 3703 & $8.6(320)$ & $2.5(92)$ \\
\hline$\geq 40$ & 658 & $7.5(49)$ & $2.3(15)$ \\
\hline \multicolumn{4}{|l|}{ Parity†: } \\
\hline 0 & 9094 & $8.8(799)$ & $2.6(237)$ \\
\hline 1 & 4960 & $7.6(376)$ & $2.0(100)$ \\
\hline 2 & 2220 & $9.1(201)$ & $2.5(55)$ \\
\hline 3 & 1091 & $8.7(95)$ & $3.0(33)$ \\
\hline$\geq 4$ & 1076 & $13.5(145)$ & $4.3(46)$ \\
\hline \multicolumn{4}{|l|}{ Timing: } \\
\hline Pre-colposcopy & 4176 & $8.0(332)$ & $2.1(88)$ \\
\hline Post-colposcopy & 14265 & $9.0(1284)$ & $2.7(383)$ \\
\hline \multicolumn{4}{|l|}{ Procedure: } \\
\hline Punch biopsy & 8999 & $7.8(705)$ & $2.2(195)$ \\
\hline LLETZ/loop/cone & 5949 & $9.2(547)$ & $3.0(178)$ \\
\hline Unknown & 3493 & $10.4(364)$ & $2.8(98)$ \\
\hline \multicolumn{4}{|l|}{ Timing $\times$ procedure } \\
\hline \multicolumn{4}{|c|}{ Birth pre-colposcopy: } \\
\hline Punch biopsy & 1736 & $6.9(119)$ & $1.8(31)$ \\
\hline Treatment & 1173 & $8.4(98)$ & $3.0(35)$ \\
\hline Unknown & 1267 & $9.1(115)$ & $1.7(22)$ \\
\hline \multicolumn{4}{|c|}{ Birth post-colposcopy: } \\
\hline Punch biopsy & 7263 & $8.1(586)$ & $2.3(164)$ \\
\hline Treatment & 4776 & $9.4(449)$ & $3.0(143)$ \\
\hline Unknown & 2226 & $11.2(249)$ & $3.4(76)$ \\
\hline Total & 18441 & $100(1616)$ & $100(471)$ \\
\hline
\end{tabular}

LLETZ=large loop excision of the transformation zone.

*Births under 33 gestational weeks.

†As recorded in hospital episode statistics, except when number of births in the dataset was greater than that recorded by hospital episode statistics. 
Table 2| Summary of analyses and results

\begin{tabular}{|c|c|c|c|c|}
\hline Analyses & Total No of births & Crude rate $(\%)$ (preterm) & Excess risk per $100(95 \% \mathrm{Cl})$ & Relative risk $(95 \% \mathrm{Cl})$ \\
\hline England & 510660 & 6.7 & - & - \\
\hline \multicolumn{5}{|l|}{ External (versus England) } \\
\hline All cohort & 18441 & 8.8 & 2.08 (1.66 to 2.49 ) & $1.31(1.25$ to 1.37$)$ \\
\hline All post-histology & 14265 & 9.0 & 2.31 (1.84 to 2.79 ) & 1.35 (1.28 to 1.42$)$ \\
\hline Post-treatment & 4776 & 9.4 & 2.71 (1.88 to 3.54$)$ & $1.41(1.29$ to 1.54$)$ \\
\hline \multicolumn{5}{|l|}{ Internal $^{*}$} \\
\hline All post-histology: & 9368 & 8.9 & - & - \\
\hline Versus all pre-histology & 3569 & 7.5 & 2.13 (0.99 to 3.27$)$ & $1.32(1.13$ to 1.53$)$ \\
\hline Post-treatment: & 3095 & 9.1 & - & - \\
\hline Versus pre-treatment & 1045 & 7.8 & 2.31 (0.34 to 4.33$)$ & $1.33(1.04$ to 1.70$)$ \\
\hline Versus post-biopsy & 4770 & 8.3 & 1.49 (0.05 to 2.95$)$ & 1.19 (1.01 to 1.41$)$ \\
\hline Adjusted & - & - & $-0.25(-2.61$ to 2.11$)$ & 0.91 (0.66 to 1.26$)$ \\
\hline \multicolumn{5}{|c|}{ Within woman/matched pairs } \\
\hline All post-histology: & 1078 & 9.1 & - & - \\
\hline Versus all pre-histology & 1078 & 7.4 & $1.67(-0.54$ to 3.88$)$ & $1.23(0.95$ to 1.59$)$ \\
\hline All treated post-histology & 372 & 8.1 & - & - \\
\hline Versus pre-histology & 372 & 8.6 & $-0.54(-4.30$ to 3.23$)$ & 0.94 (0.62 to 1.43$)$ \\
\hline
\end{tabular}

*Excess risk and relative risk adjusted by study site, parity, and maternal age at delivery. 
Table 3| Adjusted relative risks for association between cervical histology and preterm delivery

\begin{tabular}{|c|c|c|c|c|c|}
\hline \multirow[b]{2}{*}{ Variables } & \multicolumn{2}{|c|}{ Birth after colposcopy } & \multicolumn{2}{|c|}{ Birth before colposcopy } & \multirow{2}{*}{$\begin{array}{l}\text { Adjusted relative risk }{ }^{\star}(95 \% \mathrm{Cl}) \\
\text { post:pre-colposcopy }\end{array}$} \\
\hline & Total & $\%$ (No) preterm & Total & $\%$ (No) preterm & \\
\hline LLETZ/loop/cone & 3095 & $9.1(283)$ & 1045 & $81(7.8)$ & $1.33(1.04$ to 1.70$)$ \\
\hline Punch biopsy & 4770 & $8.3(396)$ & 1513 & $95(6.3)$ & $1.45(1.15$ to 1.83$)$ \\
\hline Adjusted relative risk $(95 \% \mathrm{Cl})$ cone:punch & \multicolumn{2}{|c|}{$1.19(1.01$ to 1.41$)$} & \multicolumn{2}{|c|}{$1.31(0.97$ to 1.76$)$} & $0.91(0.66$ to 1.26$)$ \\
\hline
\end{tabular}

LLETZ=large loop excision of the transformation zone.

${ }^{*}$ Adjusted by study site, parity, and maternal age at delivery. 


\section{Figures}

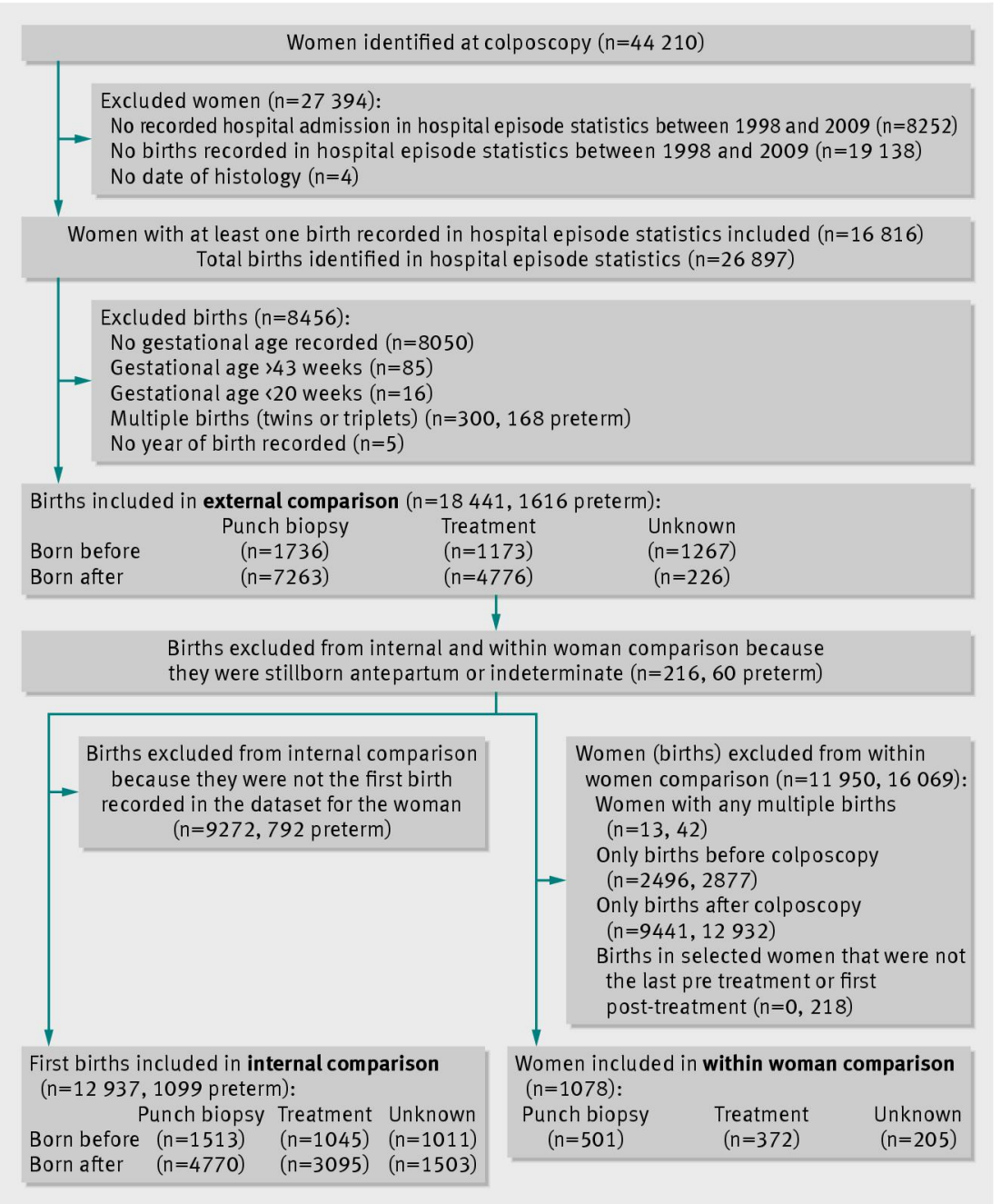

Fig 1 Flow of women through study

Study site

Wirral University Teaching Hospital

Whipps Cross University Hospital

Barts and the London NHS Trust

Royal Hallamshire Hospital

Royal Cornwall Hospital

Royal Preston Hospital

Royal Devon and Exeter NHS Foundation Trust

The James Cook University Hospital

Hammersmith Hospital, Imperial College

St James's University Hospital

North Durham County and Darlington Trust

St Mary's Hospital, Imperial College

Overall for study

England

$\begin{gathered}\text { No of total } \\ \text { births }\end{gathered}$
2608
1445
1617
1447
1529
1090
1267
1266
1148
364
272
212
14265
510660$$
0
$$

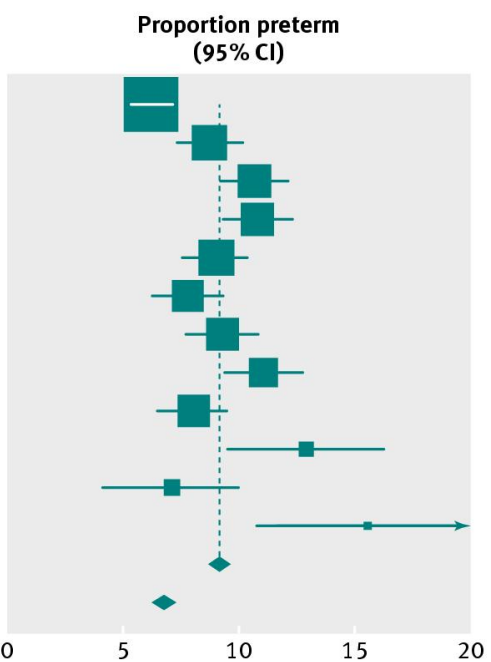

Fig 2 Proportion of preterm deliveries after colposcopy by study centre, overall, and compared with England 
Study site

Wirral University Teaching Hospital Whipps Cross University Hospital Barts and the London NHS Trust Royal Hallamshire Hospital Royal Cornwall Hospital Royal Preston Hospital Royal Devon and Exeter NHS Foundation Trust The James Cook University Hospital Hammersmith Hospital, Imperial College St James's University Hospital North Durham County and Darlington Trust St Mary's Hospital, Imperial College Overall for study

Fig 3 Relative risk of preterm birth in women with a birth after compared with before colposcopy

Weight Relative risk

(\%) $\quad(95 \% \mathrm{Cl})$

8.51 .48 (0.88 to 2.47 )

10.71 .24 (0.89 to 1.72$)$

10.10 .99 (0.69 to 1.44$)$

9.61 .68 (1.12 to 2.54$)$

$9.0 \quad 1.19$ (0.75 to 1.89$)$

$9.3 \quad 1.02$ (0.66 to 1.57 )

$7.7 \quad 1.57$ (0.83 to 2.96)

$7.1 \quad 2.41$ (1.14 to 5.09)

$7.3 \quad 1.39$ (0.68 to 2.83)

$9.3 \quad 1.29$ (0.82 to 1.99$)$

5.92 .40 (0.82 to 7.06$)$

5.50 .52 (0.15 to 1.77$)$

100.01 .32 (1.13 to 1.53 )

$\begin{array}{llllll}0.4 & 0.60 .81 & 2 & 3 & 4 & 5\end{array}$ 